\title{
Some Problems of Strength Behavior of Low-Ductile Materials at Elevated Temperatures*
}

by

\section{George S. PISARENKO**}

(Received Aug. 21, 1971)

Among the investigations that have been made at the Institute for Problems of Strength of the Academy of Sciences of the Ukrainian SSR into various issues of strength of materials and structural components operating under extreme conditions, their appreciable portion is devoted to the studies on high-temperature strength. A concise survey will be presented below of the results obtained in the investigations of the above mentioned type.

A considerable amount of interest is exhibited, in the first place, in the investigations into various aspects of the strength of refractory metals, such as tungsten, molybdenum, tantalum, niobium, and the alloys on their base. A characteristic feature of the studies on high-temperature strength of the above mentioned metals which oxidize in air at high temperatures consists in the necessity to test them in vacua.

Thus, a vacuum chamber equipped with heating devices, a vacuum system, and measuring instruments is an important element of apparatus for testing the refractory metals.

Fig. 1 shows the schematic diagram of an apparatus for investigating the major characteristic of the strength and plasticity in a vacum of $10^{-6} \mathrm{mmHg}$ at temperatures up to $3000^{\circ} \mathrm{C}$. Through a little modification of the apparatus it can be fitted to conducting its tests on creep and creep rupture strength for a time range of 2 to $60 \mathrm{~min}$.

Fig. 2 gives the results of study on the temperature dependence of tensile strength $\sigma_{b}$, and the relative elongation $\delta$, obtained for several high-temperature materials by the use of the apparatus. Fig. 3 shows the initial section of the creep curves for fibrous tungsten.

A 6-section apparatus shown in Fig. 4 is provided with a plate heater for the radiant heating of the specimens, and it has proved to be efficient for long-term studies on the creep and creep rupture strength properties in vacua at temperatures up to $3000^{\circ} \mathrm{C}$.

The stress-rupture strength curves obtained by the use of this apparatus are shown in Fig. 5 for a niobium-base alloy.

In our investigation of the fracture in brittle materials due to resistance against thermal stress, it has been found to be expedient to use hollow cylinder samples combined in a set, to be axisymmetrically heated on the internal surface by electric resistance heaters, as shown in the diagram in Fig. 6.

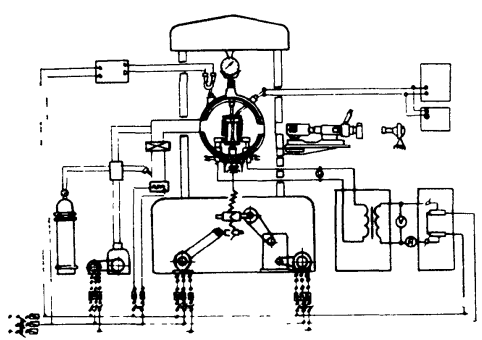

Fig. 1. Apparatus for static testing at high temperatures.
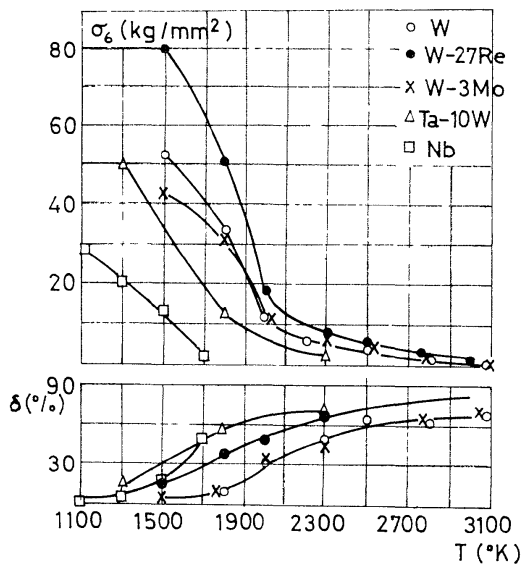

Fig. 2. Strength and plasticity curves for refractory materials.

* 原稿受理 昭和 46 年 8 月 21 日

** Institute for Problems of Strength, Academy of Sciences of the UkrSSR, Kiev, USSR. 


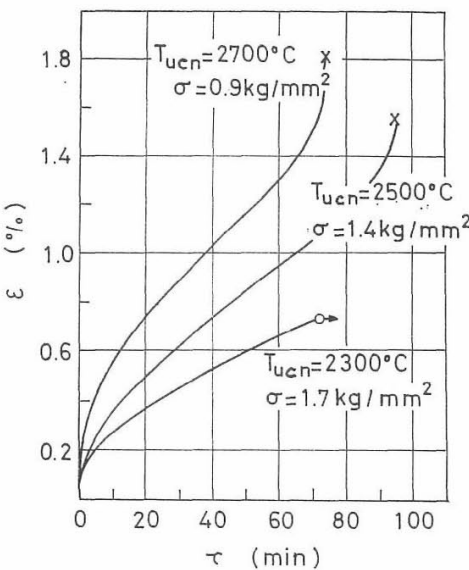

Fig. 3. Creep properties of tungsten.

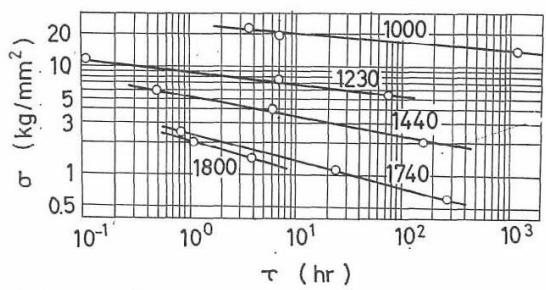

Fig. 5. Stress rupture strength curves for a niobium base alloy.

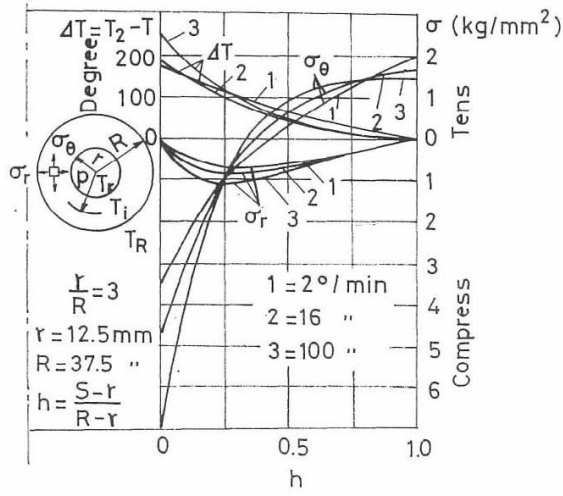

Fig. 7. Thermal-stress distributions in ring-shaped samples heated at different rates.

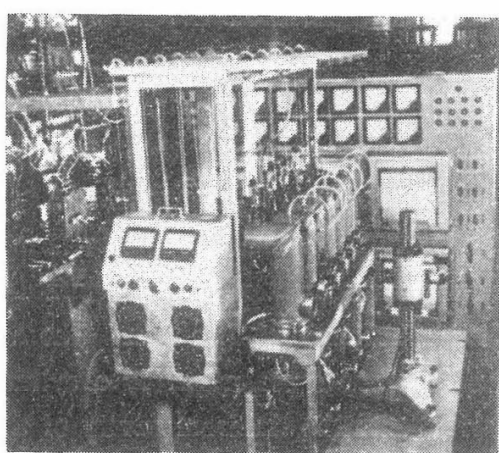

Fig. 4. Photograph of the 6-section apparatus for creep testing.

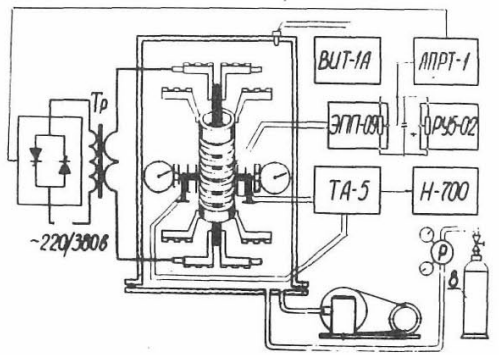

Fig. 6. Diagram of the apparatus for thermal resistance tests.

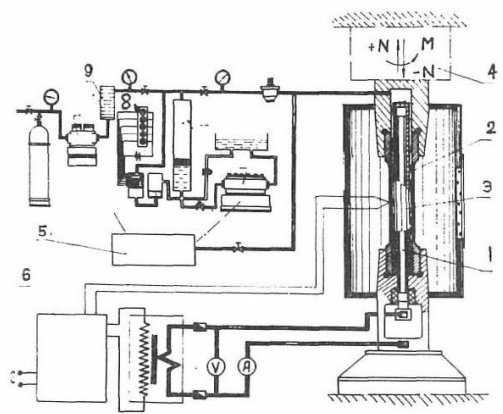

Fig. 8. Diagram of the apparatus for testing materials in the plane stress state at high temperatures.

During the test the temperature is measured continuously by thermocouples inserted in the holes which are located along the radius of the sample. Depending on the class of materials and the specified task, the temperature can be varied in the range of 20 to $2500^{\circ} \mathrm{C}$, and the heating rate in the range of 1 to $1000^{\circ} \mathrm{C}$ per min.

The apparatus works automatically, and enables us to detect and record the moment at which the fracture occurred in a given temperature field. Knowing the temperature field and thermal-physical properties of the material tested, one can quite easily compute stresses at which the fracture occurs in samples of the chosen type.

As an example, Fig. 7 shows curves of stress distribution in a ring-shaped sample of fire-clay as 
function of the temperature field conditioned by different heating rates. The obtained data suggest that failure of the sample occurs at certain approximately equal values of the relative strain in the tangential direction, produced at the butt-ends of the sample, while the relative strains in the central portion of the sample, which are generally determined through calculations, depend substantially on the height of the sample. The observed scatter of the strength indexes for inhomogeneous materials of the refractory type necessitates the use of statistical methods for processing experimental results. In particular, satisfactory solutions can be achieved by the use of the Weibull theory.

In order to study the strength criteria of heat-resistant materials in the plane stress state under high-temperature conditions, the apparatus shown on the diagram in Fig. 8 was developed at the Institute for Problems of Strength. The apparatus is designed to test a tubular sample, of which the control of heating is performed by a cylindric carborundum rod 1 protected by a thick-walled mantle 2 and inserted into the internal cavity of the sample 3. A special force exciter 4 and a controlled highpressure arrangement 5 can be applied to the programmed loading of the sample by the tensile or compressive axial load at the torsion moment under the internal pressure produced by gas medium.

To illustrate the capabilities of this apparatus, Fig. 9 gives the results of the study (on the coordinates $\sigma_{1}-\sigma_{2}$ ) on the strength behaviour of sintered metal alloy on the silicon-carbide base in the plane stress state at normal and high temperatures $\left(1020^{\circ} \mathrm{C}\right)$ with principal stress ratio ranging from 0 (uniaxial tension) to $-\infty$ (nuiaxial compression).

It will be seen from the diagram that both at normal and at high temperatures the force

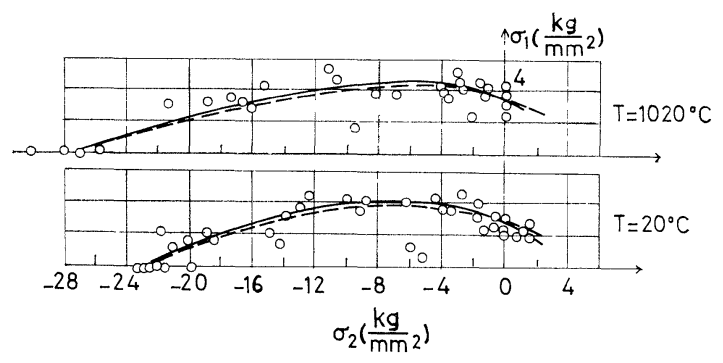

F1g. 9. Stress-ratio dependence of strength for SiC-base material.

necessary for rupture falls with increase in the transverse tensile stress, and with increase in the compressive stress, it increases up to a certain limit, which corresponds approximately to the state of pure shear.

The experimental curves indicate by nature that one of the criteria mostly used for description of limited stressed state of brittle materials is not applicable to the material in question. To evaluate the equivalent stresses a linear criterion is adopted in the form:

$$
\eta_{\Sigma}=\chi \sigma_{\imath}+(1-\chi) \sigma_{\max } P
$$

where $\sigma_{\imath}=\frac{3}{\sqrt{2}} \tau_{\text {oct }}$ is the stress intensity, $\sigma_{\max }$ is the maximum normal stress, $\chi=\frac{\sigma_{\text {ten }}}{\sigma_{\text {com }}}$ is the limited stress ratio in tension and compression, $P=A^{1-y}$ is the influence function reflecting the statistical aspects of strength in the criterion (1), $y=\frac{\sigma_{1}+\sigma_{2}+\sigma_{3}}{3}$ is the parameter of the loading severity, $A=1-\frac{1}{m}$ is a constant which depends on the material properties at a given temperature, $m$ is the homogeneity factor according to the Weibull theory.

The theoretical curves plotted by the use of the expression (1) (dashed lines) coincide practically with the experimental curves. The observed deviation may be partly explained by the fact that the values of homogeneity factor $\left(m_{20^{\circ}}=3.1 ; m_{1020^{\circ}}=4.8\right)$ have been obtained from a mass tension test of the samples belonging to another batch.

At both normal and high temperatures, the rupture of the tested samples occurs on the planes close to the principal spots on which normal tensile stresses operate, though the theory of maximum normal stresses has not been proved in the experiment.

The apparatus schematically shown in Fig. 10 was used to study the material resistance to the combined action of mechanical stress, which is not steady in time, and varying thermal stress. This apparatus can be used in experiments with cylindric specimens in the linearly stressed state with various 
combinations of both temperature and stress cycles.

Thus examining the mode of variation in temperature and mechanical-stress cycles, as shown in Fig. 11a and 11b, one can describe the ratio between the length of time of dwelling in different temperature and the stress level during the cycle of period $\tau$ under non-stationary and transitional conditions in the following form :

$$
\begin{aligned}
& \bar{\gamma}^{(T)}+\overline{\bar{\gamma}}^{(T)}+\tilde{\gamma}^{(T)}=1 ; \bar{\gamma}^{(T)}=\frac{t_{T \max }}{\tau} ; \bar{\gamma}^{(T)}=\frac{t_{T \min }}{\tau} ; \\
& \tilde{\gamma}^{(T)}=\frac{t\left({ }_{T \min } \rightleftharpoons_{T \max }\right)}{\tau} \\
& \bar{\gamma}^{(\sigma)}+\overline{\bar{\gamma}}^{(\sigma)}+\tilde{\gamma}^{(\sigma)}=1 ; \quad \bar{\gamma}^{(\sigma)}=\frac{t_{\sigma \max }}{\tau} ; \quad \bar{\gamma}^{(\sigma)}=\frac{t_{\sigma \min }}{\tau} ; \\
& \tilde{\gamma}^{(\sigma)}=\frac{t\left(T \min \rightleftharpoons_{T \max }\right)}{\tau} .
\end{aligned}
$$

Fig. 11c shows a complete space diagram of strength with various combinations of $\gamma^{(T)}$ and $\gamma^{(\sigma)}$ for $1 \mathrm{Kh} 18 \mathrm{~N} 9 \mathrm{~T}$ steel.

HF induction heating was found to be efficient in the investigation into the effect of temperature changes with wide gradient of thermal stress on fatigue behaviour of the material subjected to cycles of pure bending. For this purpose a conventional machine for testing fatigue round specimens in pure bending with rotation was provided with an induction heater which consisted of one- or two-coiled inductor embracing the gauge portion of the specimen, as shown in Fig. 12.

The use of $\mathrm{HF}$ induction heating technique with frequencies above $100 \mathrm{kc} / \mathrm{s}$ makes it possible to produce a substantially uneven distribution of temperature over the cross section of the specimen, since heat is generated only on a thin surface layer. The heater could be periodically switched on and off in accordance with a set programm, thus making it possible to study how the service life of structural components can be affected by time-variable thermal stresses often observed in actual service.

Paying attention to the study of dissipation energy in a cyclically strained material, which is regarded as a factor reducing the dynamic strain of structural elements subject to large vibrations when In service, the Institute has developed a series of testing apparatuses including a set-up for studying true energy dissipation at high temperatures under conditions of cyclic uniaxial tension-compression or pure shear. In this set-up, a sample of thin-walled tube

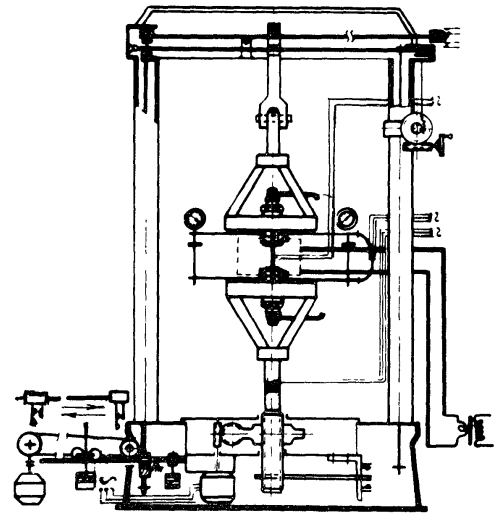

Fig. 10. Diagram of the apparatus for testing under varying conditions of both the mechanical load and the temperature.

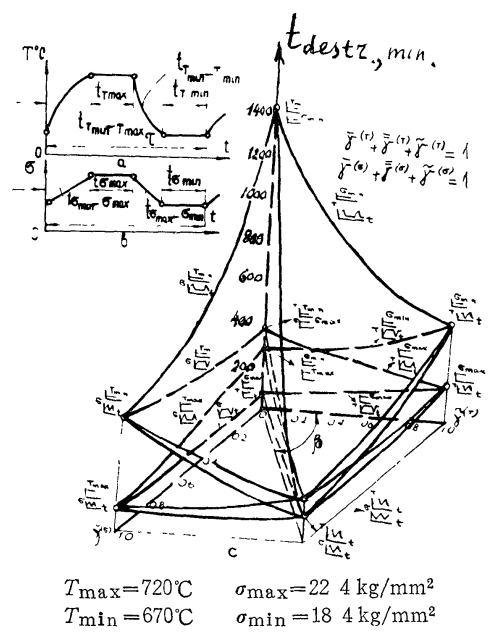

Fig. 11. Diagram of strength behaviour of steel under combined variation of mechanical load and temperature; a) temperature cycle, b) mechanical stress cycle, c) space diagram of strength.

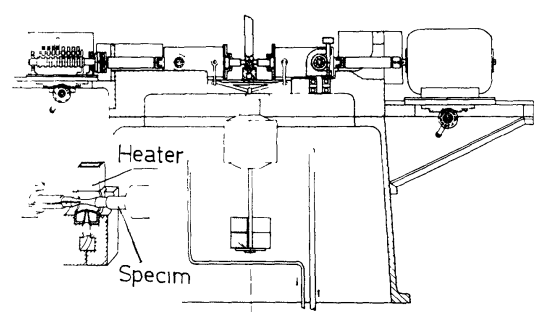

Fig. 12. Scheme of fatigue testing machine with an HF induction heater.

is heated by a rod heater inserted in the internal cavity of the sample which is fastened at its upper end in a massive disc hanging on long thin filaments. The lower end of the sample is attached to a relatively small mass fit to perform vertical and torsional oscillation in response to that of the massive disk. The oscillation system in the set-up is thus of one degree in freedom, making use of the sample under test for the spring. To prevent oxidation of both the sample and the heater, the oscillation 
system and electromagnetic excitation system of the lower mass are kept in a special chamber equipped with air-tight lead-in, a cooling system and other necessary devices.

A diagram of the above mentioned set-up is shown in Fig. 13, and the results of evaluation of oscillation decrements, obtained from vibrograms of the damping oscillations for heatresistant steels in the uniform linear stressed state are given in Fig. 14.

In discussing the investigations of the endurance of turbine blades at high temperatures under isothermal conditions and under conditions of a "soft" thermal cycling, it is important to mention the apparatus shown in Fig. 15, in which a tested blade 2 is operating as a damper of the oscillating beam 1 excited by a system of polarized magnets 3 fed from the UPB15 amplifier.

The heating of the blade (up to $900^{\circ} \mathrm{C}$ ) is performed in a furnace 4 . The stress in the blade is determined by the amplitude of the vibrations of the blade with help of a cathetometer. The loading frequency can be varied from 10 to 5000 $\mathrm{c} / \mathrm{s}$.

Fig. 16 shows a photograph of the basic part of another apparatus that is being successfully used for fatigue testing turbine blades under both isothermal and thermal-cycling conditions. In this apparatus, the moving coil of dynamic wires fed by powerful $(15 \mathrm{~kW})$ current is used for the excitation of vibrations. The heating of the blade is carried out either in an electric resistance furnace or by the use of a special inductor supplied by a HF generator.

To study the capacity of turbine discs with uneven heating in a field of centrifugal forces, the bursting rig was provided with a heating device, as shown in Fig. 17. Here the

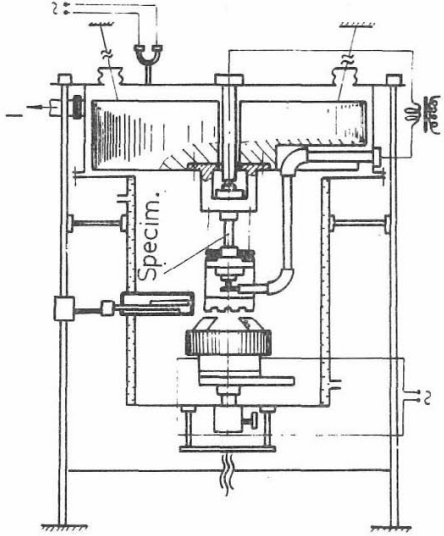

Fig. 13. Diagram of the set-up for studying the true energy dissipation.

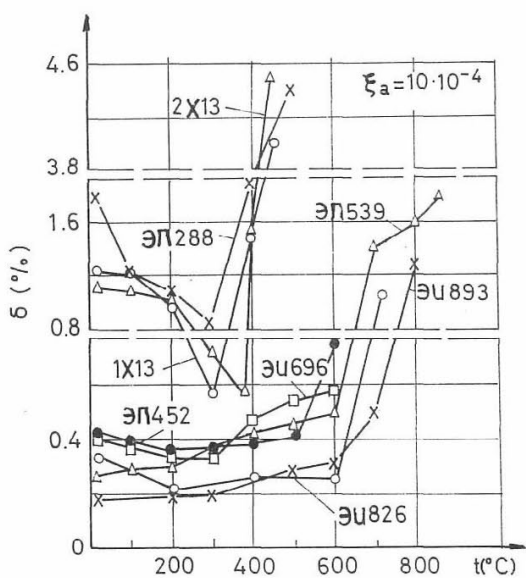

Fig. 14. Damping characteristics of some turbine-blade steels at elevated temperatures. heater is a nichrome spiral or quartz lamps. The heating zone is isolated by a thermal-insulation asbestos layer. To reduce the air resistance during the test of discs having blades, the disc arrangement along with the heater is placed in a chamber, evacuated down to $50-100 \mathrm{~mm}^{2} \mathrm{Hg}$ with help of a vacuum system.

Using the heater and a system of air-water cooling, as shown in Fig. 17, it is possible to achieve a temperature difference of 400 $500^{\circ} \mathrm{C}$ over a radius of $50 \mathrm{~mm}$ for the maximum temperature of $750^{\circ} \mathrm{C}$ as measured at the rim.

To investigate the effect of gas stream of high parameters on the load-carrying capacity of structural elements subjected to mechanical loads under both the steady and the varying

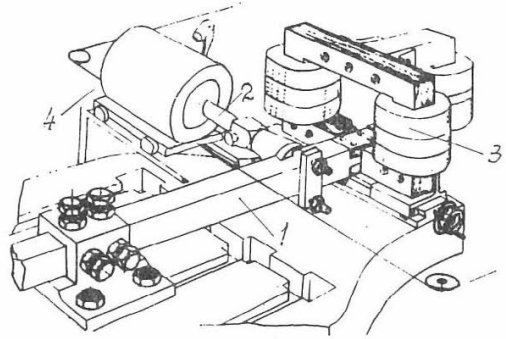

Fig. 15. Diagram of the resonance apparatus for endurance tests of turbine blades at elevated temperatures.

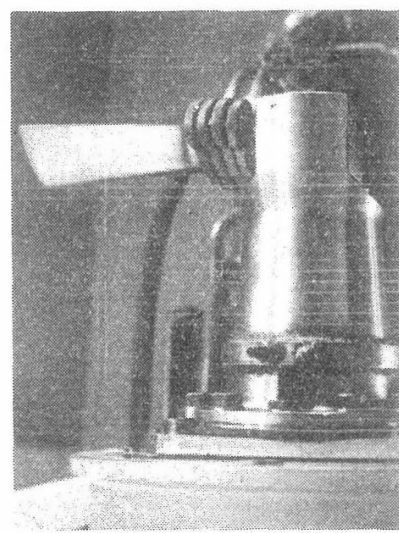

Fig. 16. Photograph of the apparatus for high-temperaturc fatigue tests of turbine blades. 
temperature conditions, a gas dynamic rig was developed at the Institute. Without dwelling on a description of the rig, since it is to be found in our monograph (Ref. 1), we should like to add only that in the rig, the temperature of the gas stream can be raised up to $2500-2800^{\circ} \mathrm{C}$, with the stream velocity being about 1 Mach.

Modifications have been made of the rig so as to make it fit for conducting, in the gas stream under isothermal conditions with temperature changes, the tests of heat-

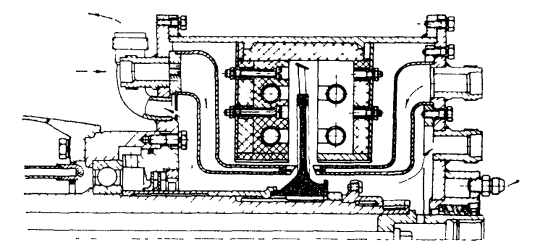

Fig. 17. Diagram of a portion of the bursting rig for testing discs under unevenheating conditions. preserving coatings, of the turbine blades with simulation of blade tension arising from centrifugal forces, and of the turbine blades under repeated-variable loads to evaluate their endurance.

In conclusion, it should be pointed out that the bounds of the present survey do not allow to discuss all new original techniques for high-temperature strength studies, developed in recent years at the Institute.

In particular, we are not able to dwell on the procedures for assessment of elastic constants at high temperatures above $3000^{\circ} \mathrm{C}$, nor for hardness tests in the same temperature range, nor on testing methods for evaluation of material endurance in high-frequency range of loading, nor on investigations into high-temperature strength of materials under neutron irradiation, etc.

Many of the above mentioned issues are elucidated in appropriate papers published regularly in the monthly "Strength of Materials" issued by our Institute, which, incidentally, is being translated into English in USA.

(Presented at the Extension Seminar on High Temperature Strength of Metals of the International Conference on Mechanical Behavior of Materials, Aug 21, 1971)

\section{References}

1) Pisarenko, G.S., V.N. Rudenko, G. N. Tretyachenko, and V.T. Troshchenko, "High-Temperature Strength of Materials (in Russian)," Naukova dumka, Kiev. (1966). 